\section{Kompass \\ Ophthalmologie}

\title{
COVID-19: Zwei Jahre Pandemie
}

Bettina Hohberger

Koordination

«OphthalmoCampus»
Liebe Leserinnen und Leser,

ein zweites Jahr der COVID-19-Pandemie geht dem Ende zu. Haben sich über den Sommer hinweg viele Erleichterungen eingestellt, so stellen die Wintermonate gerade eine Herausforderung für die Kliniken und den Privatbereich dar. Gerade hier ist es nun wichtig, dass wir alle gemeinsam zu einer Lösung für diese Situation beitragen! Und das kann jeder von uns - ob im Privaten, der Patientenversorgung oder auch im Forschungsbereich. Gerade in Letzterem hat sich in den vergangenen Monaten viel getan - und dabei sind auch wir Ophthalmologen beteiligt!

Unsere aktuelle Ausgabe beginnt sich diesem Thema zu widmen: Der erste Artikel gibt einen Einblick in die Verteilung der SARSCov2-Rezeptoren am Auge - eine grundlagenwissenschaftliche Betrachtung. Hieran schließt sich ein klinischer Blick auf die potentiellen SARS-Cov2-Auswirkungen auf die retinale Mikrozirkulation. Mit der OCT-Angiographie haben wir Augenärzte eine Methodik zur Hand, in der wir die Kapillaren nicht-invasiv darstellen können und welche die feinen Veränderungen auf Kapillarebene nach einer COVID-19-Infektion darstellen lässt. In den kommenden Ausgaben werden wir auf diese Thematik weiterführend eingehen. Es werden Personen aus verschiedenen Bereichen der Wissenschaft Einblicke bzw. einen Überblick in den Forschungsbereich zu dem Thema Long-COVID geben.

Nicht nur im Bereich COVID-19 gibt es Neuerungen - auch für die Kataraktchirurgie gibt es Neuigkeiten von IOLCon, der Roadmap zur zuverlässigen IOL-Berechnung.

Viel Freude beim Lesen dieser Ausgabe wünscht Ihre<smiles>CCCCOc1ccccc1</smiles>

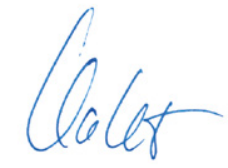

Bettina Hohberger 


\section{Aus der Forschung}

\section{Die Präsenz der SARS-CoV-2-Rezeptoren am okularen Gewebe}

Ende 2019 erschütterte der Ausbruch der Coronavirus-Krankheit 2019 (COVID-19), verursacht durch das schwere akute respiratorische Syndrom Coronavirus 2 (SARS-CoV-2) die Welt. Bis heute hat die Zahl der COVID-19-Fälle weltweit 253 Millionen überschritten, und die gemeldeten Todesfälle belaufen sich auf mehr als mehr 5,1 Millionen, mit steigender Morbidität und Mortalität (https://ourworldindata.org/ coronavirus-data). Coronaviren stellen einzelsträngige RNA-Viren dar, die Menschen und Tiere infizieren können. Über die Atemwegsmanifestationen hinaus werden systemische Schäden unterschiedlichen Schweregrades hervorgerufen, die das Gehirn, das Herz, die Nieren, das Verdauungssystem sowie die Augenoberfläche betreffen [1-3]. Das Eindringen des Coronavirus in die Wirtszellen wird hauptsächlich durch das Spike-Protein (S-Protein) vermittelt, ein Membranprotein, welches an der viralen Oberfläche exprimiert wird [4]. Das SProtein von SARS-CoV-2 bindet an seinen Wirtsrezeptor über das Angiotensin-konvertierende Enzym 2 (ACE2). Das Spike-Protein wird als inaktiver Vorläufer synthetisiert, der gespalten werden muss, um die Membranfusion zu vermitteln. Die Fusion der viralen Membran mit der Wirtszellmembran stellt den eigentlichen Eintritt des Virus in die Zelle dar und wird über die anschließende Bindung des S-Proteins an weitere Ko-Rezeptoren initiiert [5-7]. Die dadurch vermittelte Spaltung des S-Proteins in S1- und S2-Polypeptide kann durch verschiedene Korezeptoren erfolgen, darunter die Protease Furin, die
Transmembranprotease Serin 2 (TMPRSS2) oder durch CathepsinProteasen im späteren Endosom der Wirtszelle [8-10]. Speziell TMPRSS2 und Furin sind essentiell für die proteolytische Aktivierung von SARS-CoV-2 in menschlichen Atemwegszellen [16]. Durch die Spaltung wird das CendR-Motiv (C-end terminal rule) in S1 freigelegt, das wiederum an den Neuropilin-1 Rezeptor bindet [11] (Abb. 1).

Obwohl das Atmungssystem als das primäre Organ für eine SARSCoV-2-Infektion angesehen wird, haben Studien auch Zellen aus anderen Organen identifiziert, die ebenfalls mit SARS-CoV-2 infiziert werden können: Zu nennen wären Zellen aus Blutgefäßen, Nieren, Gehirn, Leber und Kardiomyozyten [12, 13]. Zum Zeitpunkt des Ausbruchs der Pandemie war wenig über die Expression der CoV-2-Rezeptoren im Auge bekannt, inzwischen gibt es zahlreiche Veröffentlichungen, die zeigen, dass die Schlüsselrezeptoren ACE2 und TMPRSS2 auf menschlichen kornealen Epithelzellen exprimiert werden und dass diese Expression auf der Augenoberfläche durch Entzündungsereignisse noch verstärkt werden kann [6, 14]. ACE2 ist ein Transmembranprotein, das auf Plasmamembranen in Arterien, Herz, Lunge und Nieren exprimiert wird. Es wurde nachgewiesen, dass ACE2 eine wichtige Rolle spielt bei der Aufrechterhaltung der Homöostase zwischen Blutdruckregulation und Angiotensin-II-Expression im Entzündungsprozess [15]. Es gibt viele Proteasen, welche Coronaviren in vitro aktivieren, darunter Furin, Cathepsin L und

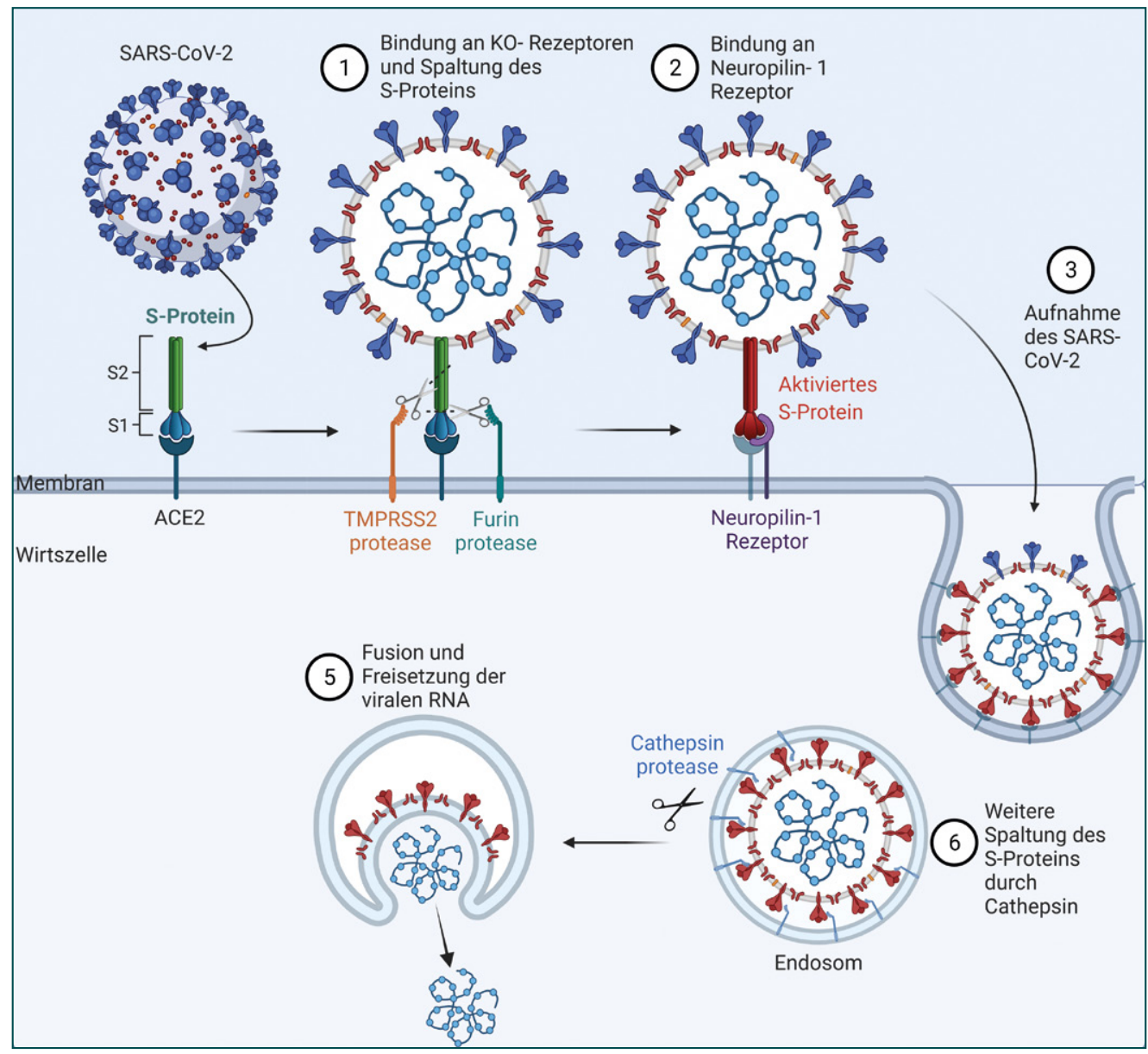

Abb. 1. Rezeptorvermittelte Aufnahme des SARS-CoV-2. Überblick der beteiligen Rezeptoren, welche die endosomale Aufnahme des Virus in die Wirtszellen vermitteln. Nach initialer Bindung an ACE2 kommt es zur Spaltung des Spike-Proteins durch die membranständigen Proteasen TMRPSS2 oder Furin [1]. Diese Spaltung - der Aktivierung des Spike-Proteins ist für die Infektion von Zellen unverzichtbar und vermittelt die Fusion zwischen Sars-Cov-2 und der Endosomenmembran. An das dadurch freigelegte Motiv kann der Neuropilin-1-Rezeptor binden, was die Infektion weiter verstärkt [2]. Nach der Internalisierung ins Endosom [3] spaltet die lysosomale Protease Cathepsin ebenfalls das Spike Protein [4], was zur Fusion und zur Freisetzung der viralen RNA ins Zytoplasma beiträgt [5]. Adapted from «Mechanism of SARS-CoV-2 Viral Entry» and «Human Coronavirus Structure» by BioRender.com (2021). Retrieved from https://app.biorender.com/biorendertemplates. 
Trypsin-ähnliche Serinproteasen, wie die TMPRSS2. Die physiologische Rolle von TMPRSS2 ist noch nicht bekannt, aber bei Mäusen mit TMPRSS2-Mangel ist kein erkennbarer Phänotyp zu beobachten, was auf eine funktionelle Redundanz schließen lässt [17]. Die Proproteinkonvertase Furin ist ein Transmembranprotein, das in eukaryontischen Geweben und Zellen ubiquitär vorkommt. Furin spaltet die Vorstufen einer breiten Palette von Proteinen, darunter Hormone, Wachstumsfaktoren, Zelloberflächenrezeptoren und Adhäsionsmoleküle, während ihres Transports entlang des sekretorischen Weges [18]. Darüber hinaus wurde Furin als aktivierende Protease für die Fusionsproteine eines breiten Viren-Spektrums identifiziert, einschließlich hochpathogener Influenza-A-Viren, HIV, Ebola-Virus, Masernvirus und Gelbfiebervirus [19]. Mitglieder der Neuropilin-Proteinfamilie haben die ungewöhnliche Fähigkeit, mit hoher Affinität an mehrere Liganden-Familien zu binden, darunter auch das S-Protein des Coronavirus. Kürzlich wurde gezeigt, dass die Bindung an Neuropilin-1 als Eintrittsfaktor in die Wirtszelle dient und die Infektiosität von SARS-CoV-2 in vitro erhöht [11, 20]. Bislang wurde der Expression und Funktion von Neuropilinen in Epithelzellen im Vergleich zu neuronalen und endothelialen Zellen wenig Aufmerksamkeit geschenkt. Neuropilin-1 ist als zelluläres Signalprotein und für seine Funktion als Zelloberflächenrezeptor für den vaskulären endothelialen Wachstumsfaktor (VEGF) und Mitglieder der SEMA3-Familie (Klasse 3 Semaphorin) bekannt [21].

Im Zuge der COVID-19-Pandemie wurde in den letzten Monaten von mehreren Forschungsgruppen die Möglichkeit einer Infektion des Auges bzw. der Hornhaut mit SARS-CoV-2 untersucht. Vor diesem Hintergrund wurde bisher vor allem die Expression des Schlüsselrezeptors ACE2 und seines Korezeptors TMPRSS2 in Epithelzellen, Hornhautgewebe, Bindehaut und Bindehautzellen untersucht. Bereits 2004 wiesen Forscher die Expression von ACE2 in menschlichen Hornhaut- und Bindehautepithelzellen nach [25]. Neuere Transkriptomanalysen bestätigten, dass die ACE2-mRNA hauptsächlich im Hornhautepithel und nicht in Keratozyten oder Endothelzellen exprimiert wird [26]. In einer weiteren Transkriptomstudie wurde die ACE2mRNA in limbalen, und in oberflächlichen konjunktivalen sowie kornealen Epithelzellen und im basalen Epithelium gefunden. In diesem Zusammenhang wurde auch die Koexpression mit TMPRSS2 dokumentiert [27]. Analysen der Proteinexpression mittels Histologie und Western-Blot aus konjunktivalem Gewebe und Zellen belegten die Kolokalisierung von ACE2 und TMPRSS2 im oberflächlichen Bindehautepithel. Allerdings gab es je nach Spender signifikante Unterschiede im Expressionsniveau [28]. Im Gegensatz dazu berichtete Lange et al., dass es keine mRNA-Expression von ACE2 und TMPRSS2 in Bindehautproben von gesunden und kranken Spendern nachgewiesen werden konnte. Auch histologisch konnte trotz zwei verschiedener Antikörper keine signifikante Expression des ACE2-Proteins detektiert werden [29]. Diese widersprüchlichen Ergebnisse in der Literatur bekräftigen auch unsere eigenen Erkenntnisse, dass erstens die Expression von ACE2 in hohem Maße von dem verwendeten Antikörper abhängt. Und zweitens, dass die Expression von SARS-CoV-2-Eintrittsfaktoren, insbesondere von ACE2, in menschlichen Augenoberflächenepithelien wahrscheinlich individuell variiert, wie dies auch für andere menschliche Gewebe vermutet wird [30]. In unserer aktuellen
Studie konnten wir die Expression von ACE2 in COVID-positiven Spenderhornhäuten in allen Epithelzellschichten nachweisen, jedoch in keiner Hornhaut von COVID-negativen Spendern. TMPRSS2 hingegen wurde nur in den oberflächlichen Epithelzellschichten, aber in allen untersuchten Hornhäuten gefunden, dasselbe galt auch für Furin und Neuropilin (Manuskript in Bearbeitung). Dazu passend berichten Li et al., dass ACE2 insbesondere in erkranktem Bindehautgewebe exprimiert wird [31]. Grundsätzlich könnten diese ungleiche Expression Grund dafür sein, der zu einer unterschiedlichen interindividuellen Anfälligkeit für SARS-CoV-2-Infektionen führt. Zusammenfassend kann festgehalten werden, dass die Hornhaut und Bindehaut alle notwendigen Voraussetzungen für eine Infektion mit SARS-CoV-2 erfüllt. Diese wird indirekt auch durch den Nachweis von SARS-CoV-2-RNA in Hornhäuten von COVID-Patienten belegt [32].

Auch in anderen Teilen des Auges wie beispielsweise im menschlichen Kammerwasser, dem Trabekelmaschenwerk, der Iris sowie im Ziliarkörper und Glaskörper von Schweineaugen konnte ACE2 nachgewiesen werden [33-35]. Zhou et al. fanden heraus, dass ACE2 und TMPRSS2 in mehreren nicht-vaskulären neuroretinalen Zellen exprimiert wurde, einschließlich der retinalen Ganglienzellschicht, der inneren plexiformen Schicht, der inneren Kernschicht und der äußeren Photorezeptorsegmente [36]. Auch in unsere Studie konnte die Expression ACE2 sowie TMPRSS2, Furin und Neuropilin in der Retina histologisch sowie auf mRNA-Ebene belegt werden (Manuskript in Bearbeitung). Damit allein würde sich die Frage, ob die menschliche Netzhaut für eine SARS-CoV-2-Infektion empfänglich ist, mit einem ja beantworten lassen. Dies wurde experimentell durch Sang et al. und Jacob et al. bestätigt. Sie zeigten anhand von pluripotenten Stammzellen (iPSC) abgeleiteten, neuralen Zellen und Organoiden an, dass das SARS-CoV-2-Pseudovirus das Nervensystem infizieren kann [37,38]. Darüber hinaus wurde SARSCoV-2 auch in postmortalen Netzhautproben von COVID-19-Patienten nachgewiesen [39].

Um weitere Schlüsse ziehen zu können, wie empfänglich ein Patient/Auge für eine SARS-CoV2-Infektion ist, reicht der alleinige Nachweis der Rezeptoren nicht aus. Man muss die Rezeptorexpression genauer analysieren, quantifizieren und die Lokalisation der Rezeptoren auf den verschiedenen Zellen mit in Betracht ziehen. Auch wurde bisher noch keine Korrelation zwischen Alter, Geschlecht, medizinscher Vorgeschichte und Rezeptorexpression gezogen.

\section{Literatur}

1. Arora R et al.: Ophthalmology 2021;128:494-503.

2. Finsterer J et al.: Clinics (Sao Paulo) 2021;76:e2900.

3. Cascella M et al.: StatPearls Publishing 2021;PMID: 32150360.

4. Yan R et al.: Science 2020;367(6485):1444-1448.

5. Hoffmann M et al.: cell 2020;181:271-280.e8.

6. Zhou L et al.: Ocul Surf 2020;18:537-544.

7. Lan J et al.: Nature 2020;581:215-220.

8. Matsuyama S et al.: J Virol 2010;84:12658-12664.

9. Simmons G et al.: Antiviral Res 2013;100:605-614.

10. Peacock TP et al.: Nature Microbiology 2021;6:899-909.

11. Daly JL et al.: Science 2020;370(6518):861-865.

12. Allison SJ: Nat Rev Nephrol 2020;16:316.

13. Yang L et al.: Cell Stem Cell. 2020;27:125-136 e7.

14. Jiang $Z$ et al.. Invest Ophthal Vis Sci. 2021;62:25.

15. Li Y et al.: Pharmacol Res 2020;157:104833.

16. Bestle D et al.: Life Sci Alliance 2020;3:e202000786. 
17. Kim TS et al.: Mol Cell Biol 2006;26:965-975

18. Garten W: Activation of Viruses by Host Proteases: Springer; 2018;205-248.

19. Böttcher-Friebertshäuser E: Activation of Viruses by Host Proteases: Springer; 2018; 153-203.

20. Cantuti-Castelvetri $L$ et al.: Science 2020;370:856-860.

21. Guo HF, Vander Kooi CW: J Biol Chem 2015;290:29120-29126.

22. Muller LJ et al.: Exp Eye Res 2003;76:521-542.

23. Gurnani B, Kaur K: Bacterial Keratitis. StatPearls. Treasure Island (FL): StatPearls Publishing LLC.; 2021.

24. Mader TH, Stulting RD: Infect Dis Clin North Am 1992;6:831-849.

25. Liu YS et al.: Chin Opthalmic Res 2004;22:561-564.

26. Sun K: bioRxiv. 2020:2020.03.30.015644.

27. Sungnak W et al.: Nat Med 2020;26:681687.

28. Xiang M et al.: Exp Eye Res 2019;184:38-47.
29. Lange C et al.: J Med Virol 2020;92:2081- 2086.

30. Bourgonje AR et al.: J Pathol 2020;251:228-248.

31. Li S et al.: Ocul Surf 2021;19:249-251.

32. Casagrande M et al.: JAMA Ophthalmol 2021;139:383-388.

33. Holappa M et al.: Open Ophthalmol J 2015;9:28-32.

34. Luhtala S et al.: J Ocul Pharmacol Ther 2009;25:23-28.

35. Ahmad Mulyadi Lai HI et al.: Int J Mol Sci 2021;22:1320.

36. Zhou L et al.: Invest Ophthalmol Vis Sci 2021;62:6.

37. Jacob F et al.: Cell Stem Cell 2020;27:937-950 e9.

38. Yi SA et al.: Viruses 2020;12:1004.

39. Casagrande M et al.: Ocul Immunol Inflamm 2020;28:721-725.

Autoren: José Hurst, Agnes Fietz, Sven Schnichels; Universitäts-Augenklinik Tübingen. Elfriede-Aulhorn-Str. 7, 72076 Tübingen, Deutschland

\section{Aus der Forschung}

\section{Das Auge als Fenster zu Long-Covid!}

Eine nicht invasive Durchblutungsmessung ist bislang nur an zwei Stellen des Körpers möglich: zum einen am Nagelfalz und zum anderen mittels OCT-A an der Retina. Bei Erkrankungen des Auges, etwa einem Glaukom, kann eine eingeschränkte Durchblutung der Retina bereits mittels OCT-A nachgewiesen werden. Wie sieht es bei systemischen Krankheiten wie Long-Covid aus?

Das SARS-CoV-2-Virus kann sich nach der Akutinfektion, weitläufig als COVID-19-Infektion bekannt, zu einer systemischen postviralen Krankheit entwickeln, die dann als «Long-Covid» beschrieben wird. Die Symptome sind vielfältig, Vorreiter sind Brain Fog, Fatigue, Konzentrationsprobleme und eine allgemein verzögerte Erholung. Das Auge betreffend berichten Patienten meist über verschwommenes Sehen. Während man dazu neigen könnte, Patienten mit dieser Symptomatik zum Neurologen zu überweisen, hat eine Forschungsgruppe der Augenklinik Erlangen nun erkundet, inwieweit die Augenheilkunde in diesen Fällen der Aufklärung dienen kann.

Als Symptome von SARS-CoV-2 wurden nicht nur pulmonale Komplikationen beschrieben, sondern auch Veränderungen des Endothels während und nach der Infektion, die wiederum möglicherweise durch die Entzündung an sich oder durch Mikrothromben verursacht worden sein können. Dies nährte den Verdacht, dass aus diesen Veränderungen auch eine verminderte Durchblutung der Retina resultieren könnte.

In der Tat wurde in einer Studie mithilfe des Spectralis II der Firma
Heidelberg Engineering gezeigt, dass Patienten, die aufgrund einer COVID-19-Infektion im Krankenhaus behandelt werden mussten, eine verminderte Mikrozirkulation in allen drei untersuchten Schichten der Makula aufwiesen. Auch Patienten, die eine wenig symptomatische oder sogar asymptomatische Akutinfektion durchgemacht hatten, zeigten im mittleren Gefäß-Plexus der Makula eine signifikante Mikrozirkulationsminderung im Vergleich zu einer gesunden Kontrollgruppe. Ebenso war die peripapilläre Mikrozirkulation aller Teilnehmer signifikant verringert.

Das OCT-A bietet einen beispielhaften nicht invasiven Einblick in das Mikrozirkulationsgeschehen des Menschen, der zwar nicht unmittelbar auf jedes Organsystem übertragbar sein mag: Dennoch zeigt die Studie, dass sich die Auswirkungen systemischer Erkrankungen damit sehr wohl zumindest unterstützend feststellen lassen. Damit also: das Auge als Fenster zur systemischen Mikrozirkulation!

\section{Literatur}

1. Hohberger B, Lucio M, Schlick S, et al.: OCT-angiography: Regional reduced macula microcirculation in ocular hypertensive and pre-perimetric glaucoma patients. PLoS ONE 2021;16:e0246469.

2. AWMF online: S1-Leitlinie Post-COVID/Long-COVID (Stand 12.07.2021). www.awmf. org/uploads/tx_szleitlinien/020-027l_S1_Post_COVID_Long_COVID_2021-07.pdf

3. Verdecchia P, Cavallini C, Spanevello A, Angeli F: The pivotal link between ACE2 deficiency and SARS-CoV-2 infection. Eur J Intern Med. 2020;76:14-20.

Autor: Jakob Hoffmanns, Augenklinik, Universitätsklinikum Erlangen, recover.au@uk-erlangen.de

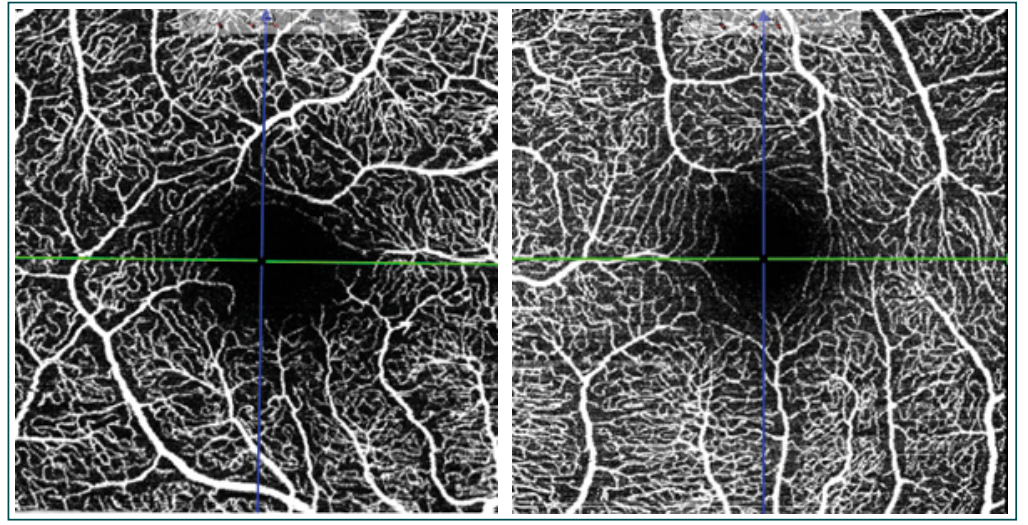

Abb. 1. Proband nach einer Covid-19 Infektion (li.), Proband ohne CovidInfektion (re).. 


\section{Ophthalmologie kompakt}

\section{Neuigkeiten von IOLCon, der Roadmap zur zuverlässigen IOL-Berechnung}

Sie sind aus der Berechnung von Intraokularlinsen (IOL) heute nicht mehr wegzudenken, weil sie das refraktive Ergebnis nach der Katarakt-OP wesentlich verbessern können: optimierte IOL-Kontanten, am besten individuell optimierte. Die 2017 gegründete Internet-Datenbank IOLCon (www.iolcon.org) hat sich inzwischen als weltweit verfügbare, zuverlässige Quelle für optimierte IOL-Konstanten und -Spezifikationen etabliert. Basierend auf zeitgemäßen Optimierungsstrategien stellt IOLCon auch individuell optimierte IOL-Konstanten Ophthalmo-Chirurgen kostenfrei zur Verfügung. Das von IOLCon angewandte Verfahren zur Optimierung der IOLKonstanten ist gekennzeichnet als eine «Intelligente IOL-Konstanten-Optimierung», das z.B. statistische Verfahren unter Berücksichtigung der Messgenauigkeiten der Messinstrumente (z.B. Biometer) verwendet. Auf IOLCon sind jeweils Optimierungen der Konstanten für folgende veröffentlichte Formeln zu finden: Haigis, Hoffer-Q, Holladay 1, SRK/T - und jetzt auch für die junge CastropFormel.

Mit der Castrop-Fomel steht seit Kurzem eine neue Formel zur Berechnung der IOL-Stärke zur Verfügung, bei der die Position der IOL anhand realer historischer Messdaten regressiv vorhergesagt wird

(basierend auf ca. 2000 Datensätze). Mehr Informationen zur Castrop-Formel, die bereits auch als torische Variante zur Verfügung steht, wurden im Open-Access-Verfahren kürzlich veröffentlicht:

- Langenbucher, et al.: Considerations on the Castrop formula for calculation of intraocularlens power.PLoS One. 202116(6):e0252102. www.ncbi.n/m.nih.gov/pmc/articles/PMC8172026

- Langenbucher, et al.: The Castrop formula for calculation of toric intraocular lenses. Graefes Arch Clin Exp Ophthalmol. 2021; 259(11): 3321-3331. www.ncbi.n/m.nih.gov/pmc/articles/PMC8523386

Voraussetzung ist die Nutzung von aktuellen Messtechniken, die alle Teilstrecken des Auges genau vermessen. Mit diesen Daten und in Verbindung mit modernen Formeln (wie der Castrop-Formel) sind auch Augen, die außerhalb der Norm liegen und die bei Nutzung klassischer Formeln Schwierigkeiten machen, zuverlässiger zu berechnen.

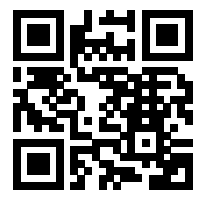

Mehr Info auf https://iolcon.org.

Kontakt: Univ.-Prof. Dr. Achim Langenbucher, Achim.Langenbucher@uks.eu 